\title{
Effects of osteoblast autophagy on glucocorticoid-induced femoral head necrosis
}

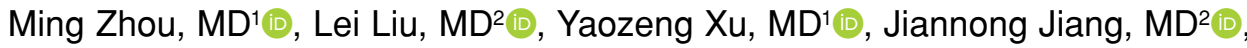 \\ Gang Liu, $\mathrm{MD}^{3}$ (D), Chenjun Zhai, $\mathrm{MD}^{2}$ (i)
}

'Department of Orthopedics, First Affiliated Hospital of Soochow University, Suzhou, Jiangsu Province, People's Republic of China ${ }^{2}$ Department of Orthopedics, Affiliated Yixing Hospital of Jiangsu University, Yixing, Jiangsu Province, People's Republic of China ${ }^{3}$ Department of Orthopedics, Second Affiliated Hospital of Xuzhou Medical University, Xuzhou, Jiangsu Province, People's Republic of China

Femoral head necrosis (FHN) is a pathological process typified by the death of bone active components. ${ }^{[1-3]}$ With the aggravation of hormonal drug abuse, steroid-induced avascular necrosis of the femoral head (SANFH) has become a common bone and joint disease clinically, which usually endangers bilateral femoral heads with a wide range of osteonecrosis at the end stage. Because of the high disability rate after onset, most patients eventually choose hip replacement surgery.

In recent years, autophagy has been closely related to many aging and degenerative diseases, including aging-induced systemic bone loss. Autophagy is a self-eating behavior widely existing in cells, as an intracellular process transporting substances to lysosomes for degradation. ${ }^{[4]}$ Like apoptosis and

Received: February 07, 2020

Accepted: May 05, 2020

Published online: September 11, 2020

Correspondence: Yaozeng Xu, MD. Department of Orthopedics, First Affiliated Hospital of Soochow University, Suzhou 215006. Jiangsu Province, People's Republic of China.

E-mail: xuyzfahsu@aliyun.com

Doi: $10.5606 /$ ehc. 2020.73036

Citation: Zhou M, Liu L, Xu Y, Jiang J, Liu G, Zhai C. Effects of osteoblast autophagy on glucocorticoid-induced femoral head necrosis. Jt Dis Relat Surg 2020;31(3):411-418.

(2020 All right reserved by the Turkish Joint Diseases Foundation

This is an open access article under the terms of the Creative Commons Attribution-NonCommercial License, which permits use, distribution and reproduction in any medium, provided the original work is properly cited and is not used for commercial purposes (http://creativecommons.org/licenses/by-nc/4.0/).

\section{ABSTRACT}

Objectives: This study aims to explore the mechanism by which osteoblast autophagy participated in glucocorticoid-induced femoral head necrosis (FHN).

Materials and methods: Thirty male specific-pathogen-free C57 mice (age, one month; weighing 20-25 g) were randomly divided into blank control, dexamethasone and rapamycin-dexamethasone groups $(n=10)$. After six weeks of intervention, right femoral head was obtained to observe morphology and to calculate percentage of empty lacunae. MC3T3-E1 cells were randomly divided into normal, dexamethasone, rapamycin and dexamethasone-rapamycin groups, and cultured for $24 \mathrm{~h}$. Microtubule-associated protein 1 light chain 3 (LC3)-I, LC3-II, mammalian target of rapamycin (mTOR) and Beclin-1 protein expressions were detected by Western blot.

Results: In rapamycin-dexamethasone group, some bone trabeculae in medullary cavity ruptured and atrophied, and subchondral bone underwent local necrosis. The total apoptosis rates of dexamethasone and rapamycin-dexamethasone groups surpassed that of blank control group, and the former two groups had significantly different rates $(\mathrm{p}<0.001)$. LC3-II/LC3-I of dexamethasone group was lower than those of rapamycin and dexamethasone-rapamycin groups $(\mathrm{p}<0.001)$, and the ratio of rapamycin group surpassed that of dexamethasone-rapamycin group $(p<0.001)$. Dexamethasone group had higher mTOR protein expression than those of rapamycin and dexamethasonerapamycin groups $(\mathrm{p}<0.001)$, and the expression of rapamycin group was lower than that of dexamethasone-rapamycin group $(\mathrm{p}<0.001)$. The Beclin-1 protein expression of dexamethasone group was lower than those of rapamycin and dexamethasonerapamycin groups $(\mathrm{p}<0.001)$, and the expression of rapamycin group exceeded that of dexamethasone-rapamycin group $(\mathrm{p}<0.05)$.

Conclusion: Osteoblast autophagy may play a crucial protective role in dexamethasone-induced FHN. The attenuation of autophagy may be related to the affected expressions of key autophagy regulators mTOR and Beclin-1.

Keywords: Autophagy, femoral head, glucocorticoid, necrosis, osteoblast. 
endoplasmic reticulum stress, autophagy is also a crucial physiological phenomenon in cells relying on a highly conserved autophagy process in each species to buffer the stress response of the external environment, to degrade harmful organelles, and to maintain normal metabolism and homeostasis of the internal environment.

Recently, the important roles of autophagy in human health and diseases have been highlighted, and the protective effects of autophagy under various stresses have been proven, ${ }^{[5]}$ suggesting that the changes of autophagy activity dominate in the prevention and development of diseases. The influence of autophagy on bone metabolism is well documented. For instance, Liu et al. ${ }^{[6]}$ found that autophagy was activated during the differentiation of osteoblasts. Besides, Zhang et al. ${ }^{[7]}$ reported that the expressions of various autophagy-related genes were associated with bone density. However, they failed to determine whether autophagy exerted beneficial or adverse effects. Autophagy is of great significance to the maintenance of mature bone formation..$^{[8]}$ During aging, osteoblast function is weakened and bone loss is affected by the progressive damage to osteoblast autophagy. ${ }^{[9]}$ In high glucose environments, autophagy is a crucial mechanism for osteoblast protection. ${ }^{[10]}$ At present, autophagy is a well-established protective mechanism of cartilage itself. The autophagy level of chondrocytes gradually decreases along with aging, thus being one of the important reasons for articular cartilage degeneration..$^{[11-14]}$

However, it remains controversial whether the autophagy level changes in the procession of hormoneinduced FHN. Microtubule-associated protein 1 light chain 3 (LC3) is a key gene encoding autophagyrelated proteins which are localized on the surfaces of pre-autophagic vesicles and autophagic vesicle membranes. It plays an essential role in the processing and modification of two ubiquitin-like proteins, and participates in autophagosome formation. Beclin- 1 is a homologue of yeast autophagy-related 6 (Atg6) protein. Beclin-1 autophagy-related proteins are localized on the pre-autophagosome to bind phosphatidylinositol3-kinase (PI3K), forming a Beclin-1-Vps34-Vps15 core complex which plays a vital role in the early stage of autophagosome formation. Luo and Rubinsztein ${ }^{[15]}$ reported that Beclin-1 overexpression in HeLa cells caused a continuous increase in the number of intracellular autophagosomes. Additionally, Qu et al. ${ }^{[16]}$ found that Beclin-1-deficient mice underwent significantly reduced autophagic vacuole formation, suggesting that the regulation of Beclin-1 expression was consistent with autophagy. As a conserved serine/threonine protein kinase, mammalian target of rapamycin (mTOR) facilitates cell autophagy when its activity is inhibited, and suppresses autophagy when its activity is enhanced. ${ }^{[17]}$ In this study, we aimed to explore the mechanism by which osteoblast autophagy participated in glucocorticoid-induced FHN.

\section{MATERIALS AND METHODS}

This study was conducted at First Affiliated Hospital of Soochow University between November 2018 and November 2019. Thirty male, specific-pathogen-free and healthy $\mathrm{C} 57$ mice (age, one month; weighing 20-25 g) were purchased from Shanghai Jiao Tong University School of Medicine [animal license number: SCXK (Shanghai) 2018-0007]. MC3T3-E1 was obtained from American Type Culture Collection, USA.

Rapamycin was purchased from Sigma (Sigma Corp., St. Louis, MO, USA). Dexamethasone sodium phosphate injection was bought from Guangdong Sancai Shiqi Pharmaceutical Co., Ltd. (Guangzhou, China). Cell Counting Kit-8 (CCK8), Radio Immunoprecipitation Assay (RIPA) cell lysate, bicinchoninic acid (BCA) protein assay reagent and SDS-PAGE gel preparation kit were obtained from Beyotime Institute of Biotechnology Co., Ltd. (Shanghai, China). Minimum Essential Medium Eagle - alpha modification ( $\alpha$-MEM) was provided by Thermo Fisher Scientific (Suzhou) Instruments Co., Ltd. (Soochow, China). Hematoxylin and eosin (HE) staining kit was purchased from Beijing Solarbio Science \& Technology Co., Ltd. (Beijing, China). mTOR antibody, LC3 B antibody, Beclin-1 antibody and $\beta$-actin antibody were bought from Cell Signal Technology (Danvers, MA, USA). Enhanced chemiluminescence (ECL) reagent was obtained from Invitrogen (Carlsbad, CA, USA). Polyvinylidene fluoride (PVDF) membrane and ultrapure water purification system were provided by Millipore (Burlington, MA, USA).

Thirty mice were randomly divided into blank control, dexamethasone and rapamycindexamethasone groups $(n=10)$. The blank control group was intraperitoneally injected with $5 \mathrm{mg} \bullet \mathrm{kg}^{-1}$ rapamycin-free solvent. The dexamethasone group was intraperitoneally injected with $2.5 \mathrm{mg} \bullet \mathrm{kg}^{-1}$ dexamethasone sodium phosphate injection and $5 \mathrm{mg} \bullet \mathrm{kg}^{-1}$ rapamycin-free solvent. The rapamycindexamethasone group was intraperitoneally injected with $2.5 \mathrm{mg} \bullet \mathrm{kg}^{-1}$ dexamethasone sodium phosphate injection and $5 \mathrm{mg} \bullet \mathrm{kg}^{-1}$ rapamycin. After six weeks of intervention, the mice were euthanized, from which 
the skin and subcutaneous tissue were cut from the right thigh, and muscles around the femur were disconnected. Finally, the complete right femoral head was obtained, fixed in $10 \%$ formaldehyde solution, decalcified in $10 \%$ ethylenediaminetetraacetic acid solution, paraffin-embedded and sectioned.

The right femoral head was scanned with micro-computed tomography (CT) to observe its morphology. The sections were placed in a $55^{\circ} \mathrm{C}$ oven for $30 \mathrm{~min}$, infiltrated in xylene for $10 \mathrm{~min}$ and refreshed xylene for another $10 \mathrm{~min}$, rinsed three times with phosphate-buffered saline (PBS) (five min each time), hydrated with absolute ethanol and gradient concentrations of ethanol solutions rinsed three times with PBS (five min each time) and stained with hematoxylin for $10 \mathrm{~min}$. After excess staining solution was washed off with clean water for $10 \mathrm{~min}$, the sections were washed with distilled water for $10 \mathrm{sec}$, discolored and differentiated with $1 \%$ hydrochloric acid-ethanol for $5 \mathrm{sec}$, stained with eosin for about one min, rinsed three times with PBS (five min each time) and then dehydrated with gradient concentrations of ethanol solutions. After dehydration was completed, the sections were transparentized with xylene for five min and mounted with neutral resin to observe the morphology of the femoral head under an optical microscope. Five fields of view were selected under high magnification, and 50 bone lacunae were counted in each field to calculate the percentage of empty lacunae, which was used to indicate the overall apoptosis rate. Percentage of empty lacunae $=$ number of empty lacunae/number of bone lacunae $\times 100 \%$.
The relative growth rate of MC3T3-E1 cells was detected by CCK 8 assay. After passage in $\alpha$-MEM to the third generation, the cells were counted, diluted and seeded into a 96-well plate at a density of $5 \times 10^{4} /$ well. After cell adherence, the culture medium was discarded. Then the cells were added $\alpha$-MEM containing dexamethasone at $0 \mathrm{mM}$ (control), 10-8 $\mathrm{M}$, 10-6 $\mathrm{M}$ or 10-4 M, and incubated for $24 \mathrm{~h}$. Afterwards, $10 \mu \mathrm{L}$ of CCK8 solution was added to each well, and the plate was placed in an incubator at $37^{\circ} \mathrm{C}$ for another one $\mathrm{h}$. The 96-well plate was taken out from the incubator, and the optical density (OD) was measured by a microplate reader at $450 \mathrm{~nm}$ to calculate the relative growth rate. Relative cell growth rate $=\left(\mathrm{OD}_{\text {experimental group }}-\mathrm{OD}_{\text {medium }}\right) /\left(\mathrm{OD}_{\text {control group }}\right.$ - $\left.\mathrm{OD}_{\text {medium}}\right)$.

According to the optimal pre-experimental results (dexamethasone concentration: 10-6 M; incubation time: $24 \mathrm{~h}$ ), the third-generation MC3T3-E1 cells were randomly divided into four groups, and cultured with $\alpha$-MEM (normal group), $\alpha$-MEM containing 10-6 M dexamethasone (dexamethasone group), $\alpha$-MEM containing $100 \mathrm{nM}$ rapamycin (rapamycin group), and $\alpha$-MEM containing 10-6 M dexamethasone and $100 \mathrm{nM}$ rapamycin (rapamycin-dexamethasone group) for $24 \mathrm{~h}$.

MC3T3-E1 cells were lysed with $200 \mu \mathrm{L}$ of precooled RIPA lysis buffer, and centrifuged at $4^{\circ} \mathrm{C}$ and $12,000 \mathrm{rpm}$ for five min to collect the supernatant. Total protein concentration was measured by the BCA method. Subsequently, proteins were separated by $5 \%$ SDS-PAGE, and the products were electronically transferred onto a PVDF membrane. The membrane was thereafter washed three times with Tris buffered
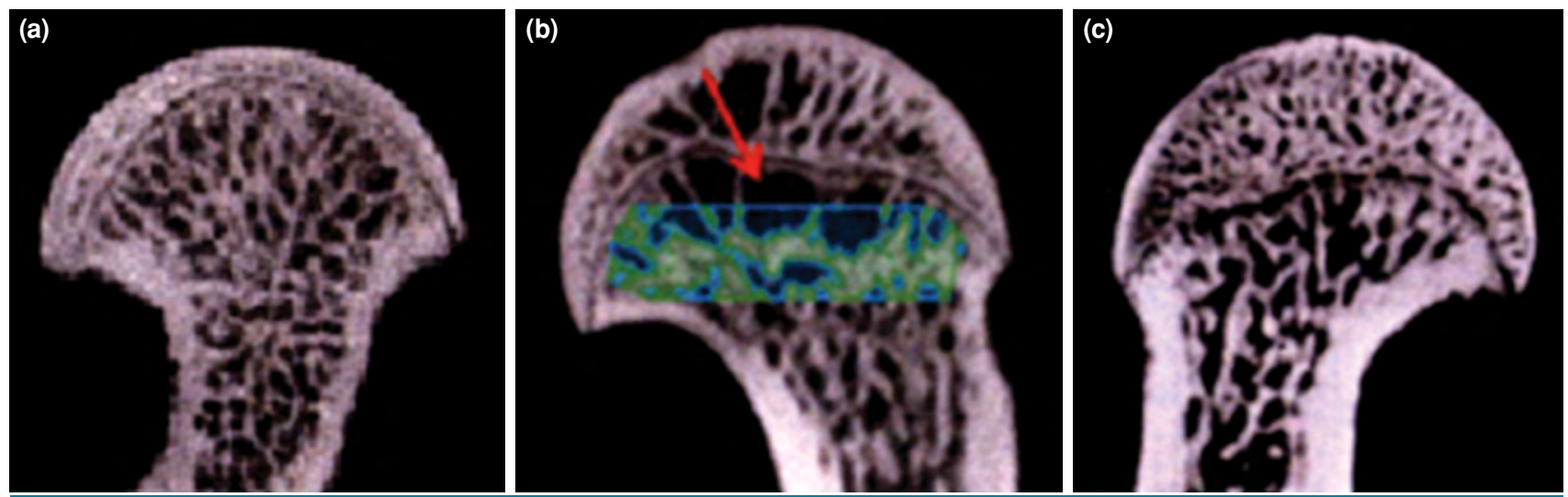

FIGURE 1. Computed tomography scan results of femoral heads. (a) Blank control group. (b) Dexamethasone group. (c) Rapamycin-dexamethasone group.

Red arrow: Fracture. 

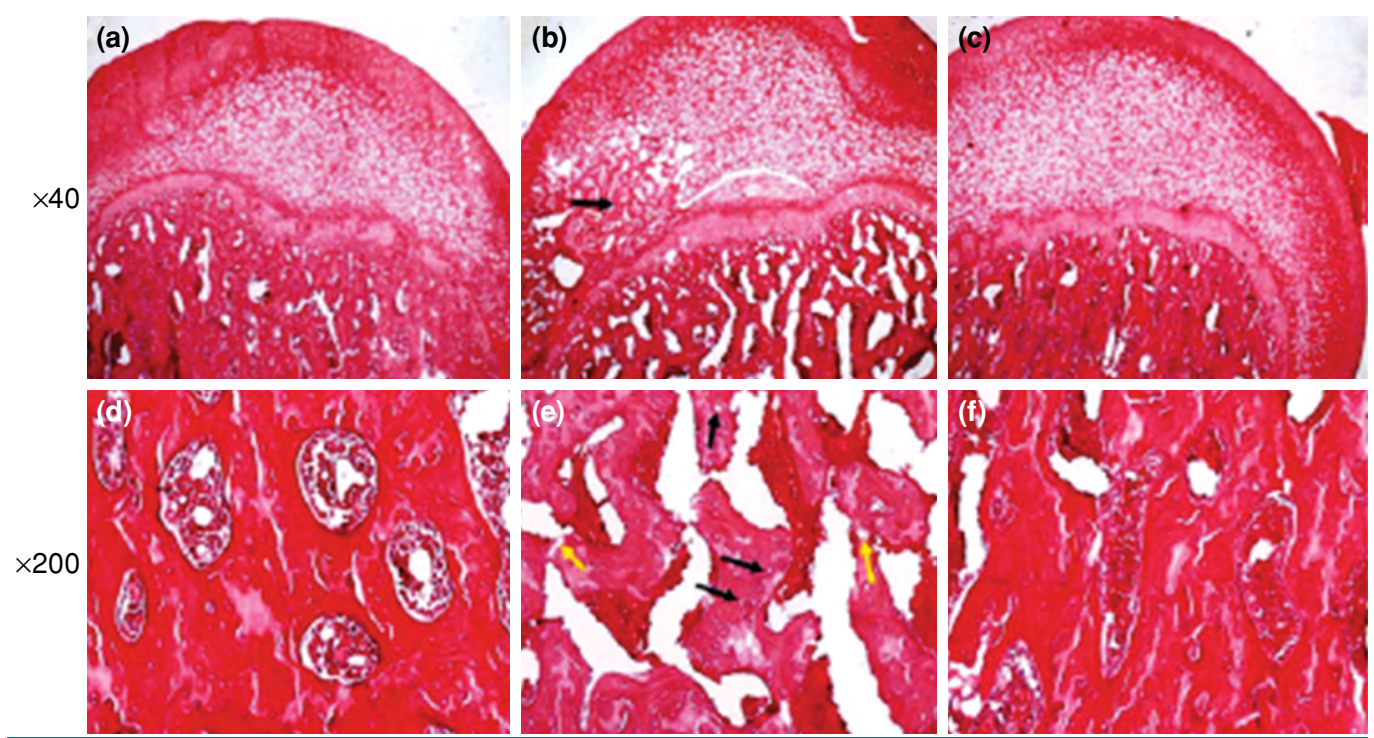

FIGURE 2. Microscopic observation of histomorphology of femoral heads. (a, d) Blank control group; (b, e) Dexamethasone group; (c, f) Rapamycin-dexamethasone group.

Yellow arrow: Trabecular fracture; Black arrow: Empty bone lacuna.

saline with Tween 20 (TBST) (10 min each time), blocked in the blocking solution at room temperature for one $h$, incubated with primary antibodies against LC3B, mTOR and Beclin-1 (1:300 diluted) and $\beta$-actin (1:1,000 diluted) overnight at $4^{\circ} \mathrm{C}$, washed, incubated with secondary antibodies against at room temperature in dark for $60 \mathrm{~min}$, and washed by TBST. Afterwards, the membrane was developed by using ECL reagent, and the gray values of protein bands were analyzed by Image Lab (Image Lab LLC, Sarasota, FL, USA) software.

\section{Statistical analysis}

All data were statistically analyzed by IBM SPSS version 20.0 software (IBM Corp., Armonk, NY, USA). Comparisons among different groups were performed by one-way analysis of variance, and pairwise intergroup comparisons were conducted with the least significant difference (LSD)-t test. $\mathrm{P}<0.05$ was considered statistically significant.

\section{RESULTS}

The CT scan showed that in the blank control group, the trabeculae of the femoral head were in good shape and evenly distributed, without the formation of subchondral necrosis zone (Figure 1a). In the dexamethasone group, the subchondral necrosis zone of the femoral head was formed, the trabeculae were occluded, and that in the medullary cavity was thin, sparse, fractured, and the cortical bone was thickened (Figure 1b). In the rapamycin-dexamethasone group, the CT imaging findings of the femoral head were between the dexamethasone group and the blank control group. The fracture and atrophy of the trabeculae in the medullary cavity and the partial necrosis of the subchondral bone were observed (Figure 1c).

Microscopy showed that the trabeculae of the femoral head in the blank control group were in good shape, without empty bone lacunae, adipocyte

\begin{tabular}{|lccc|}
\hline \multicolumn{4}{c|}{ TABLE I } \\
& Total cell apoptosis rate & & \\
\hline Group & Apoptosis rate (\%) & F value & $p$ \\
\hline Blank control & $6.3 \pm 0.8$ & 208.187 & $<0.001$ \\
Dexamethasone & $64.0 \pm 4.4^{*}$ & & \\
Rapamycin-dexamethasone & $33.3 \pm 3.3^{*}$,\# & \\
\hline * Comparison between blank control group and other two groups, $p<0.001$; \# Comparison \\
between dexamethasone group and rapamycin-dexamethasone group, $p<0.001$.
\end{tabular}




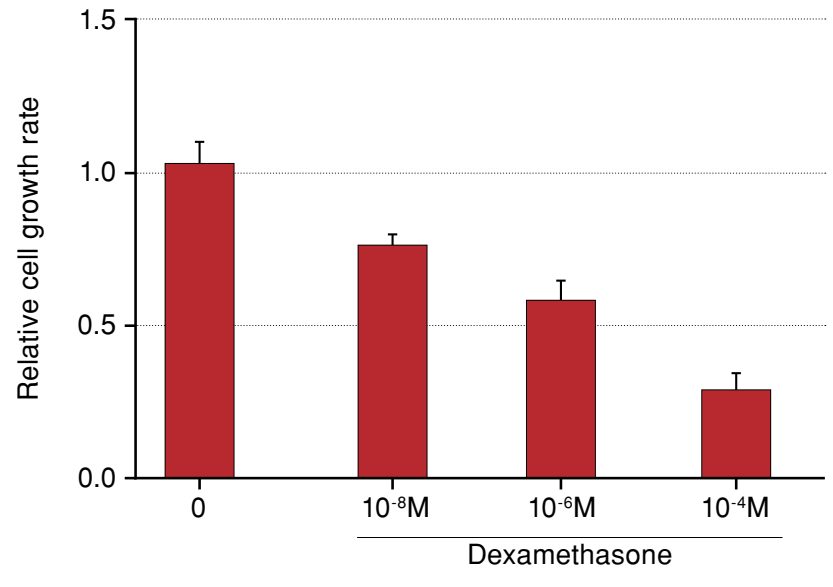

FIGURE 3. Cell growth after $24 \mathrm{~h}$ of treatment with different concentrations of dexamethasone. and lipid deposition, or tissue necrosis and fibrosis (Figure 2a and $\mathrm{d}$ ). The trabeculae of the femoral head in the dexamethasone group were sparse and disordered, the number of adipocytes increased, the lipid deposited in the bone cells, and a large number of empty bone lacunae were formed, with local tissue necrosis, fibrosis and decreased cell number (Figure $2 b$ and e). In the rapamycin dexamethasone group, some of the trabeculae of the femoral head were fractured and atrophied, and a small amount of empty bone lacunae were observed (Figure 2c and f).

The total apoptosis rates of the dexamethasone group $(64.0 \pm 4.4 \%)$ and the rapamycin-dexamethasone group $(33.3 \pm 3.3 \%)$ were significantly higher than


FIGURE 4. Microtubule-associated protein 1 light chain 3-I//microtubule-associated protein 1 light chain 3-I ratio and protein expressions of mammalian target of rapamycin and Beclin-1.

LC: Microtubule-associated protein 1 light chain; mTOR: Mammalian target of rapamycin. 
that of the blank control group $(6.3 \pm 0.8 \%)(p<0.001)$, and the former two groups also had significantly different rates $(\mathrm{p}<0.001)$ (Table $\mathrm{I})$.

The growth rate of MC3T3-E1 cells untreated with dexamethasone exceeded those of the cells treated with different concentrations of dexamethasone for $24 \mathrm{~h}(\mathrm{p}<0.001)$ (Figure 3).

The LC3-II/LC3-I ratio of the normal group was higher than that of the dexamethasone group $(\mathrm{p}<0.001)$, lower than that of the rapamycin group $(\mathrm{p}<0.001)$, and similar to that of the dexamethasonerapamycin group $(p>0.05)$. LC3-II/LC3-I of the dexamethasone group was lower than those of rapamycin and dexamethasone-rapamycin groups $(\mathrm{p}<0.001)$. The rapamycin group had higher LC3-II/ LC3-I than that of the dexamethasone-rapamycin group $(\mathrm{p}<0.001)$. The $\mathrm{mTOR}$ protein expression of the normal group was lower than that of the dexamethasone group $(\mathrm{p}<0.05)$, and higher than those of rapamycin and dexamethasone-rapamycin groups $(p<0.001)$. The dexamethasone group had higher mTOR protein expression than those of rapamycin and dexamethasone-rapamycin groups $(\mathrm{p}<0.001)$. The mTOR protein expression of the rapamycin group was lower than that of the dexamethasonerapamycin group $(\mathrm{p}<0.001)$. The Beclin-1 protein expression of the normal group was higher than that of the dexamethasone group, but similar to those of rapamycin and dexamethasone-rapamycin groups ( $p>0.05)$. The Beclin-1 protein expression of the dexamethasone group was lower than those of rapamycin and dexamethasone-rapamycin groups $(\mathrm{p}<0.001)$. The rapamycin group had higher Beclin-1 protein expression than that of the dexamethasonerapamycin group $(\mathrm{p}<0.05)$ (Figure 4$)$.

\section{DISCUSSION}

The pathogenesis of FHN is the interruption or damage of blood supply to the femoral head, leading to apoptosis of bone cells. ${ }^{[18]}$ At present, there are many theories on the pathogenesis of hormonal FHN, such as osteoporosis, intraosseous hypertension, intravascular coagulation, osteotoxin effect, osteocyte apoptosis, and fat metabolism disorder, ${ }^{[1-3]}$ but its exact pathogenesis remains unclear.

Autophagy is an important protective mechanism of cartilage itself, and the reduction of autophagy is considered to be one of the important causes of articular cartilage degeneration. Zhang et al. ${ }^{[19]}$ found that mesenchymal stem cells can promote their survival by increasing the level of autophagy in hypoxia and serum-free conditions. Besides,
Gao et al. ${ }^{[20]}$ reported that autophagy also played a key protective role in the process of hormoneinduced degeneration and apoptosis of bone cells by in vitro bone cell culture, and the number of bone cell death after autophagy significantly increased. In this study, a steroid-induced FHN model was established by intraperitoneal injection of $2.5 \mathrm{mg} \bullet \mathrm{kg}^{-1}$ dexamethasone sodium phosphate. After the model was established, the femoral head tissue obtained was stained with $\mathrm{HE}$, and the morphology of the femoral head was observed under a microscope. The results showed that under the intervention of dexamethasone, the adipocytes in femoral head of the mice increased, the lipid deposited in the bone cells, the trabeculae of the femoral head were sparse and disordered, and the number of empty bone lacunae increased in the dexamethasone group, which was more than that of the blank control group and the dexamethasone and rapamycin group. According to the results of micro$\mathrm{CT}$, in the mouse hormone FHN model constructed by dexamethasone alone, subchondral necrosis zone of femoral head was formed, trabeculae were occluded, trabeculae in medullary cavity became thin, sparse and fractured in the medullary cavity, and thickened bone cortex appeared. However, after intraperitoneal injection of rapamycin, the femoral head was in good shape, some trabeculae were fractured and atrophied, and the subchondral necrosis zone was significantly less than that of the dexamethasone-constructed hormonal FHN mice.

In vitro, Western blot was used to detect the expression of autophagy-related proteins mTOR, Beclin-1 and LC3 in MC3T3-E1 cells. The results showed that the ratio of LC3-II/LC3-I and the content of Beclin-1 protein in MC3T3-E1 cells of the dexamethasone group were significantly lower than those of other groups, while the expression of mTOR protein was significantly higher than that of other groups. The ratio of LC3-II/LC3-I in MC3T3-E1 cells in the dexamethasone-rapamycin group was significantly higher than that of the dexamethasone group, and the expression of mTOR protein was lower than that of the dexamethasone group. Therefore, dexamethasone stimulation alone can reduce the level of autophagy, while rapamycin can antagonize the inhibition of autophagy. Li et al. ${ }^{[21]}$ explored the mechanism underlying dexamethasone-induced osteoblast injury, and found that $24 \mathrm{~h}$ of treatment with 1 and $10 \mu \mathrm{mol} / \mathrm{L}$ dexamethasone significantly up-regulated Beclin-1 and LC3 expressions $(\mathrm{p}<0.05)$. The study of Li et al. ${ }^{[21]}$ showed that dexamethasone significantly promoted autophagy, and that 
overactivated autophagy may be a crucial factor contributing to SANFH. In contrast, we herein found that dexamethasone suppressed autophagy, which may be one of the causes of FHN. The inconsistent results may be attributed to different dexamethasone doses. Meanwhile, cell autophagy may be a doubleedged sword. In other words, either overactivated or decreased autophagy has a negative effect, finally inducing hormonal FHN.

Moreover, Liao et al. ${ }^{[22]}$ reported that compared with the control group, the LC3-II/LC3-I ratio of dexamethasone group gradually decreased from $12 \mathrm{~h}$ $(\mathrm{p}<0.01)$ to $24 \mathrm{~h}(\mathrm{p}<0.05)$, reaching the minimum at $48 \mathrm{~h}$. They verified that autophagy was inhibited by dexamethasone treatment time-dependently. Furthermore, Luo et al. ${ }^{[23]}$ reviewed the role of autophagy in steroid-induced FHN. They concluded that autophagy was a self-protection mechanism for FHN, and autophagy and apoptosis participated in the pathogenesis of steroid-induced FHN, with the level of autophagy associated with the dose of hormone.

The LC3 is a recognized autophagy marker. When autophagy is formed, cytosolic LC3 (i.e. LC3-I) can hydrolyze a small segment of polypeptide and transform into an autophagosome model (i.e. LC3-II). The LC3-II/LC3-I ratio can be used to estimate the level of autophagy. ${ }^{[24]}$ The mTOR complex and Beclin-1 complex are key molecules regulating autophagy in cells, which play a crucial role in the development of autophagy. ${ }^{[25,26]}$ It is generally accepted that mTOR plays a regulatory role in autophagy through two mechanisms: (i) mTOR-mediated signal transduction acts on downstream effectors, such as transcription initiation factor $4 \mathrm{E}$ binding protein 1 and ribosomal protein S6 kinase, and initiates related gene transcription and translation to control autophagy; (ii) mTOR kinase acts directly on Atg protein to regulate autophagosome formation. ${ }^{[27,28]}$ Autophagy is promoted when mTOR activity is inhibited, and suppressed when mTOR activity is increased. Beclin-1, also known as BECN1, is a homolog of the yeast autophagy protein 6 (ATG6) gene and a specific gene of mammals involved in autophagy. Beclin-1 gene regulates the localization of other Atg proteins in the structure of autophagy precursor and regulates autophagy activity by forming complex with type III PI3K. ${ }^{[2,30]}$ It is well established that up-regulation of Beclin-1 expression in mammalian cells can stimulate the occurrence of autophagy, but it cannot happen after knockings out this gene. ${ }^{[25,28]}$

Rapamycin used in this study is a classical autophagy enhancer, and the regulation pathway of autophagy mainly involves the classical PI3K/protein kinase $\mathrm{B} /$ rapamycin target protein signaling pathway. However, rapamycin can induce and promote autophagy by inhibiting mTOR, and the influence of many factors on autophagy is related to the change of mTOR activity. ${ }^{[31,32]}$ Herein, after rapamycin enhanced autophagy level, the apoptosis level and cell growth inhibition rate decreased significantly. It indicates that autophagy can inhibit the death of MC3T3-E1 cells stimulated by dexamethasone to a certain extent, and autophagy plays a protective role on osteoblasts (MC3T3-E1), which will provide a new idea for the treatment of hormonal FHN.

The number of used animals is limited. Besides, the findings must be further verified by clinical studies.

In conclusion, autophagy plays a crucial protective role in the pathogenesis of hormonal FHN. The decrease of autophagy level induced by dexamethasone may be one of the causes of FHN, and its mechanism may be related to the expression of mTOR and Beclin-1, which are the key regulators of autophagy stimulated by dexamethasone. In the future, the improvement of autophagy level can be an important target for the prevention or treatment of hormonal FHN.

\section{Declaration of conflicting interests}

The authors declared no conflicts of interest with respect to the authorship and/or publication of this article.

\section{Funding}

The authors received no financial support for the research and/or authorship of this article.

\section{REFERENCES}

1. Haberal B, Şahin O, Şimşek EK, Mahmuti A, Tuncay İC. Outcomes for core decompression with multiple drilling of the osteonecrosis of the femoral head in patients with solid organ transplantation. Eklem Hastalik Cerrahisi 2018;29:159-64.

2 Maurel DB, Boisseau N, Benhamou CL, Jaffre C. Alcohol and bone: review of dose effects and mechanisms. Osteoporos Int 2012;23:1-16.

3. Kang JS, Park S, Song JH, Jung YY, Cho MR, Rhyu $\mathrm{KH}$. Prevalence of osteonecrosis of the femoral head: a nationwide epidemiologic analysis in Korea. J Arthroplasty 2009;24:1178-83.

4. Yang Z, Klionsky DJ. Eaten alive: a history of macroautophagy. Nat Cell Biol 2010;12:814-22.

5. Kroemer G, Mariño G, Levine B. Autophagy and the integrated stress response. Mol Cell 2010;40:280-93.

6. Liu F, Fang F, Yuan H, Yang D, Chen Y, Williams L, et al. Suppression of autophagy by FIP200 deletion leads to osteopenia in mice through the inhibition of osteoblast terminal differentiation. J Bone Miner Res 2013;28:2414-30. 
7. Zhang L, Guo YF, Liu YZ, Liu YJ, Xiong DH, Liu XG, et al. Pathway-based genome-wide association analysis identified the importance of regulation-of-autophagy pathway for ultradistal radius BMD. J Bone Miner Res 2010;25:1572-80.

8. Manolagas SC, Parfitt AM. What old means to bone. Trends Endocrinol Metab 2010;21:369-74.

9. Weinstein RS, Wan C, Liu Q, Wang Y, Almeida M, O'Brien $\mathrm{CA}$, et al. Endogenous glucocorticoids decrease skeletal angiogenesis, vascularity, hydration, and strength in aged mice. Aging Cell 2010;9:147-61.

10. Bartolomé A, López-Herradón A, Portal-Núñez S, GarcíaAguilar A, Esbrit P, Benito M, Guillén C. Autophagy impairment aggravates the inhibitory effects of high glucose on osteoblast viability and function. Biochem J 2013;455:329-37.

11. Caramés B, Hasegawa A, Taniguchi N, Miyaki S, Blanco FJ, Lotz M. Autophagy activation by rapamycin reduces severity of experimental osteoarthritis. Ann Rheum Dis 2012;71:575-81.

12. Lotz MK, Caramés B. Autophagy and cartilage homeostasis mechanisms in joint health, aging and OA. Nat Rev Rheumatol 2011;7:579-87.

13. Sasaki H, Takayama K, Matsushita T, Ishida K, Kubo S, Matsumoto T, et al. Autophagy modulates osteoarthritisrelated gene expression in human chondrocytes. Arthritis Rheum 2012;64:1920-8.

14. Yang RT, Zhang C, Liu Y, Zhou HH, Li ZB. Autophagy prior to chondrocyte cell death during the degeneration of Meckel's cartilage. Anat Rec (Hoboken) 2012;295:734-41.

15. Luo S, Rubinsztein DC. Apoptosis blocks Beclin 1-dependent autophagosome synthesis: an effect rescued by Bcl-xL. Cell Death Differ 2010;17:268-77.

16. Qu X, Yu J, Bhagat G, Furuya N, Hibshoosh H, Troxel A, et al. Promotion of tumorigenesis by heterozygous disruption of the beclin 1 autophagy gene. J Clin Invest 2003;112:1809-20.

17. Wang F, Shan S, Huo Y, Xie Z, Fang Y, Qi Z, et al. MiR155-5p inhibits PDK1 and promotes autophagy via the mTOR pathway in cervical cancer. Int J Biochem Cell Biol 2018;99:91-9.

18. Pouya F, Kerachian MA. Avascular Necrosis of the Femoral Head: Are Any Genes Involved? Arch Bone Jt Surg 2015;3:149-55.

19. Zhang Q, Yang YJ, Wang H, Dong QT, Wang TJ, Qian HY, et al. Autophagy activation: a novel mechanism of atorvastatin to protect mesenchymal stem cells from hypoxia and serum deprivation via AMP-activated protein kinase/mammalian target of rapamycin pathway. Stem Cells Dev 2012;21:1321-32.

20. Gao J, Cheng TS, Qin A, Pavlos NJ, Wang T, Song Ket al. Glucocorticoid impairs cell-cell communication by autophagy-mediated degradation of connexin 43 in osteocytes. Oncotarget 2016;7:26966-78.

21. Li X, Li YS, Li LJ, Xie X, Yang Y, Deng ZH, et al. Overactivated autophagy contributes to steroid-induced avascular necrosis of the femoral head. Exp Ther Med 2017;14:367-72.

22. Liao Y, Zhang P, Yuan B, Li L, Bao S. Pravastatin Protects Against Avascular Necrosis of Femoral Head via Autophagy. Front Physiol 2018;9:307.

23. Luo P, Gao F, Han J, Sun W, Li Z. The role of autophagy in steroid necrosis of the femoral head: a comprehensive research review. Int Orthop 2018;42:1747-53.

24. Fritzen AM, Frøsig C, Jeppesen J, Jensen TE, Lundsgaard AM, Serup AK, et al. Role of AMPK in regulation of LC3 lipidation as a marker of autophagy in skeletal muscle. Cell Signal 2016;28:663-74.

25. Pattingre S, Espert L, Biard-Piechaczyk M, Codogno P. Regulation of macroautophagy by mTOR and Beclin 1 complexes. Biochimie 2008;90:313-23.

26. Eskelinen EL. New insights into the mechanisms of macroautophagy in mammalian cells. Int Rev Cell Mol Biol 2008;266:207-47.

27. Scott RC, Schuldiner O, Neufeld TP. Role and regulation of starvation-induced autophagy in the Drosophila fat body. Dev Cell 2004;7:167-78.

28. Rosenfeldt MT, Ryan KM. The role of autophagy in tumour development and cancer therapy. Expert Rev Mol Med 2009;11:e36.

29. Scarlatti F, Maffei R, Beau I, Codogno P, Ghidoni R. Role of non-canonical Beclin 1-independent autophagy in cell death induced by resveratrol in human breast cancer cells. Cell Death Differ 2008;15:1318-29.

30. Gaytán M, Morales C, Sánchez-Criado JE, Gaytán F. Immunolocalization of beclin 1, a bcl-2-binding, autophagy-related protein, in the human ovary: possible relation to life span of corpus luteum. Cell Tissue Res 2008;331:509-17.

31. Korolchuk VI, Saiki S, Lichtenberg M, Siddiqi FH, Roberts EA, Imarisio S, et al. Lysosomal positioning coordinates cellular nutrient responses. Nat Cell Biol 2011;13:453-60.

32. Poüs C, Codogno P. Lysosome positioning coordinates mTORC1 activity and autophagy.Nat Cell Biol 2011;13:342-4. 